\title{
Intermittent claudication after radiotherapy for testicular malignancy
}

\author{
Claudia Cruceru ${ }^{1}$, Roxana Enache ${ }^{1,2}$, M. Simion ${ }^{1}$, Andreea Călin ${ }^{1,2}$, Sorin Băilă ${ }^{3}$, Dan Deleanu ${ }^{4}$, \\ Liliana Parascan ${ }^{5}$, Carmen Ginghină ${ }^{1,2}$
}

'Department of Cardiology,

"Prof. Dr. C. C. lliescu" Emergency Institute of Cardiovascular Diseases, Bucharest, Romania

2"Carol Davila” University of Medicine and Pharmacy, Bucharest, Romania

"Department of Vascular Surgery,

"Prof. Dr. C. C. lliescu" Emergency Institute of Cardiovascular Diseases, Bucharest, Romania ${ }^{4}$ Department of Cardiac Catheterization,

"Prof. Dr. C. C. lliescu" Emergency Institute of Cardiovascular Diseases, Bucharest, Romania ${ }^{5}$ Department of Pathology,

"Prof. Dr. C. C. lliescu” Emergency Institute of Cardiovascular Diseases, Bucharest, Romania

\author{
Corresponding author: \\ Roxana Enache, MD \\ "Carol Davila" University of Medicine \\ and Pharmacy, Sos. Fundeni, \\ No 258, 022328, Bucharest, Romania \\ E-mail: roxenache@gmail.com
}

\section{ABSTRACT}

The most common cause of intermitent claudication in males is atherosclerosis involving the arteries of the lower limbs. Irradiation represents a major risk factor for development of arterial lesions, its association with conventional cardiovascular risk factors increasing even more the likelihood of vascular damage. We present the case of a 39-year-old patient diagnosed with peripheral arterial disease 8 years after radiotherapy for testicular cancer. Revascularization surgery was performed. It seemed that the arterial involvement had a double etiology, both radiation-induced and atherosclerotic.

Key words: femoral artery, arterial injury, radiotherapy, testicular cancer

\section{INTRODUCTION}

Radiation arteritis remains an underdiagnosed pathology, despite the prolonged survival of the cancer patients $(1,2)$. Although there are no large studies, cardiovascular complications following radiotherapy were described in up to $40 \%$ of patients (1). However, the prevalence of large vessels injuries after irradiation remains difficult to assess. As for radiation treated testicular tumors, there are only a few cases of lower limb arterial damage reported (3).

\section{CASE REPORT}

We report the case of a 39-year-old patient who was admitted to hospital for intermittent claudication in the right calf and tingling in the right leg, with 
gradually reduced walking threshold in the last 6 months, at present experiencing pain at more than 200 $\mathrm{m}$. The pain was associated with great efforts and was relieved by rest. The patient was a current smoker (smoking 1 package of cigarettes daily for 20 years), had been diagnosed with grade 3 hypertension and dyslipidemia 3 years ago, and had a family history of cardiac ischemic disease. His past medical history included 20 sessions of right inguinal radiotherapy for a surgically treated testicular cancer 8 years ago. Physical examination revealed cyanosis of the right foot's fingertips and no pulse in dorsalis pedis arteries and right posterior tibial artery. Laboratory studies showed hypertriglyceridemia ( $302 \mathrm{mg} / \mathrm{dl}$ ). Thrombophilia tests revealed normal values. Echocardiographic evaluation was normal. Right ankle-brachial index was 0.58 . Doppler ultrasound examination of lower limb revealed a $90 \%$ stenosis of the right superficial femoral artery at $1 \mathrm{~cm}$ from its origin, of about $1 \mathrm{~cm}$ length with intraluminal hypoechogenic material (figure 1). Downstream, right popliteal artery and right tibial trunks had slow poststenotic flow.

Lower limb arteriography revealed a short subocclusion at the origin of superficial femoral artery with possible thrombus features, occlusion at the origin of deep femoral artery (figure 2) and bilateral occlusion of anterior tibial and peroneal arteries. Given the history of early age onset hypertension, a renal angiogram was performed which revealed normal renal arteries. After multidisciplinary consultation it was decided to proceed with surgical revascularization due to existence of lesions on both superficial femoral artery and deep femoral artery. Thrombendarterectomy of right common and superficial femoral artery was performed and a vein graft was applied (figures 3, 4, 5). At the level of the deep femoral artery, thrombembolectomy using a Fogarty catheter was performed.

Histopathologic examination of the resected material revealed vascular fibro-collagenous tissue with an area of repairing granulation tissue, foam cells and a recent thrombus consisting of fibrin, erythrocytes and leukocytes (figures 6, 7). The postoperative course was uneventful. The patient was discharged receiving treatment with oral anticoagulation, aspirin, statin, angiotensin receptor blocker and gastric protection.

\section{DISCUSSIONS}

As arterial lesions continue to be an underestimated consequence of irradiation, the pathophysiological mechanism of these injuries remains unclear. It might be a radiation specific molecular response, or just a classic

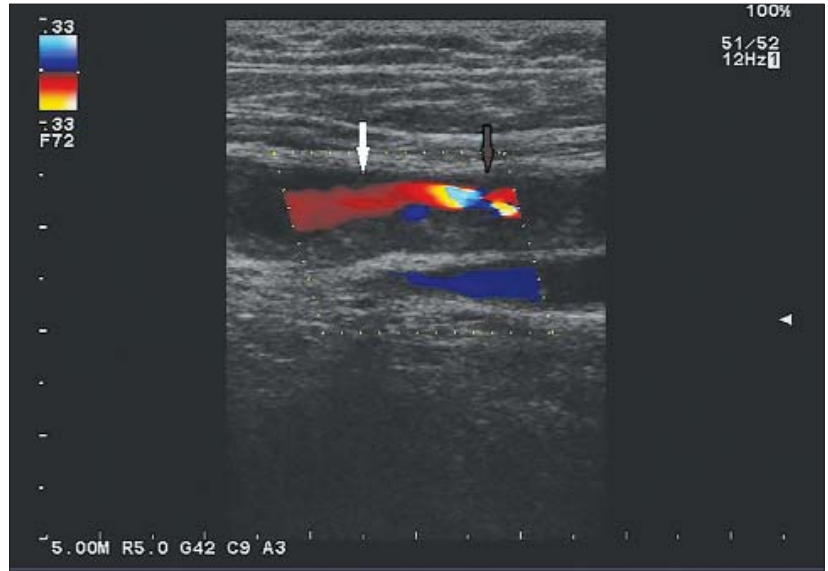

Figure 1 - Color Doppler examination of the right superficial femoral artery (white arrow) showing severe stenosis (gray arrow) of about $1 \mathrm{~cm}$ length

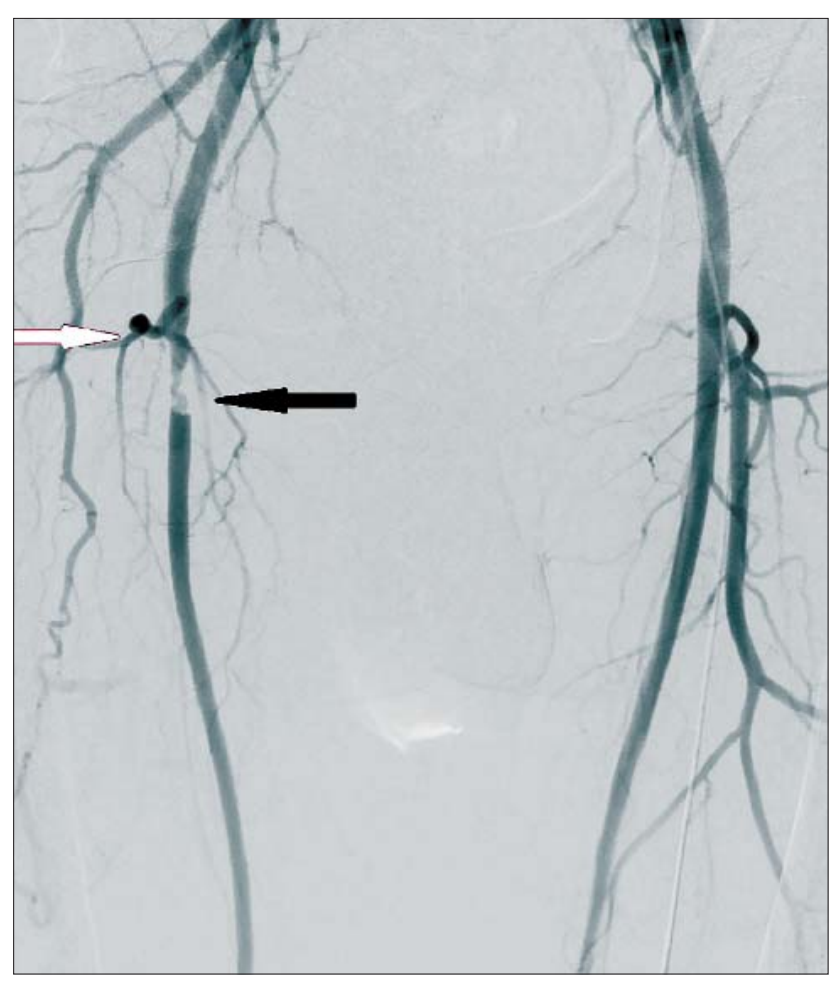

Figure 2 - Lower limb arteriography showing arterial subocclusion at the origin of superficial femoral artery (black arrow) and occlusion at the origin of deep femoral artery (white arrow)

accelerated atherosclerotic process (1).

Used to treat cancerous cells, radiotherapy produces injuries in the adjoining healthy tissues, affecting both small and large vessels in different ways. It seems that the small arteries are more radiosensitive than the large arteries; at this level there is evidence of inflammation that leads to endothelial dysfunction and then to a prothrombotic and antifibrinolytic response $(1,2)$. In addition, oxidative stress resulted from radiolysis of water contributes to oxidation of low-density lipo- 


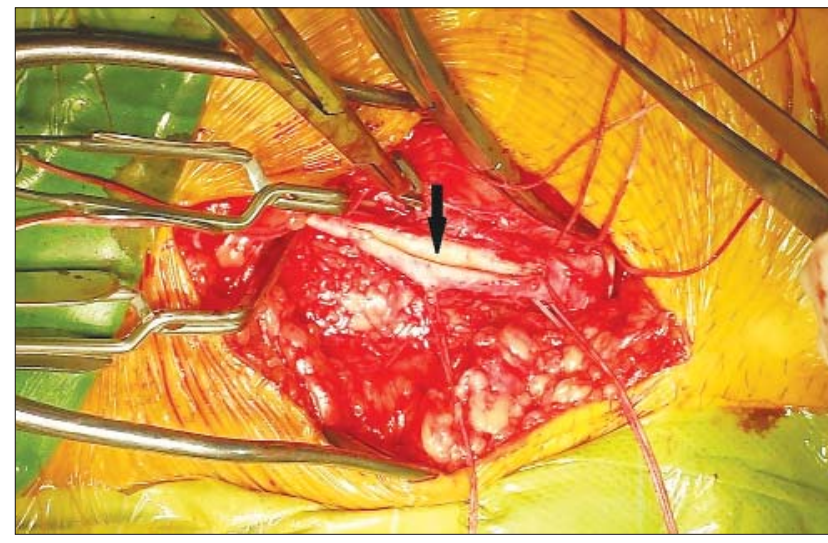

Figure 3 - Operative view of the dissected right superficial femoral artery showing atherom plaques (arrow)

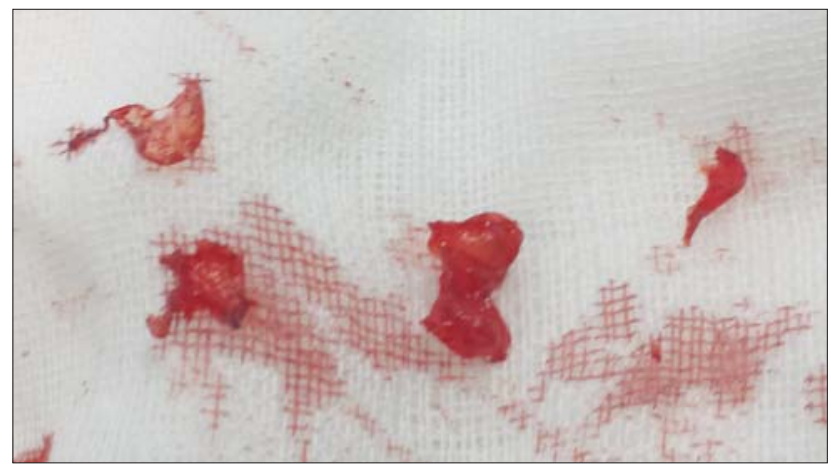

Figure 5 - Macroscopic view of the specimen

proteins, recruiting leukocytes that will become lipidladen foam cells and inducing expression of inflammatory adhesion molecules $(2,4)$. Regarding cytokines and growth factors, an important role in this process is played by IL-1 $\beta$ and transformation growth factor $\beta$ (TGF- $\beta$ ), which helps fibroblasts become fibrocytes $(2,5)$. Radiotherapy can impair telomeres, producing senescence to the cells (4).

Changes that can appear in an irradiated artery include intimal proliferation and thickening, smooth muscle cell hypertrophy and collagen deposition, disruption of elastic lamina, fibrosis, and obliteration of the vasa vasorum (6). In large vessels as femoral artery, the first changes may include myointimal proliferation, thrombosis and rupture, while arterial fibrosis, stenosis and accelerated local atherosclerosis appear later $(7,8)$. These arteries are less often affected than the smaller vessels, due to their large lumen and thick wall, made of relatively radioresistant cells (9). These changes leading to the thickening of the endothelium and fibrosis induce the narrowing of the arterial lumen, favoring thrombus formation, embolization, or occlusion (10).

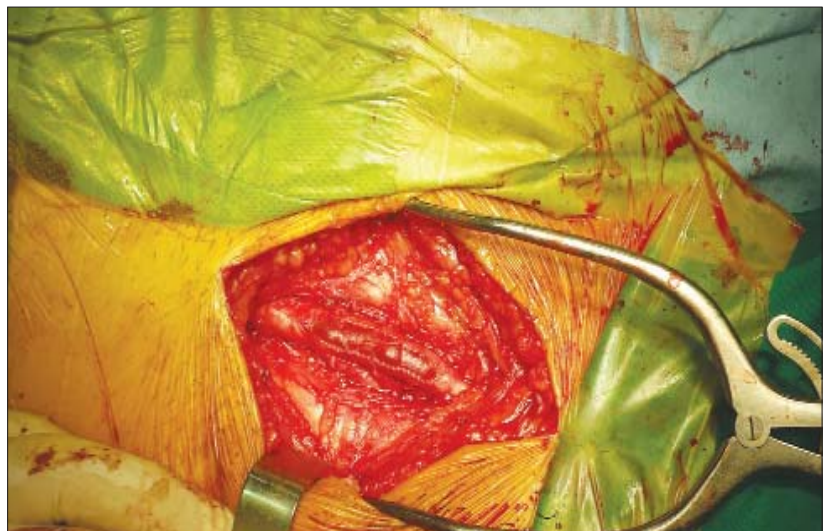

Figure 4 - Operative view of the right superficial femoral artery reconstruction with a vein graft

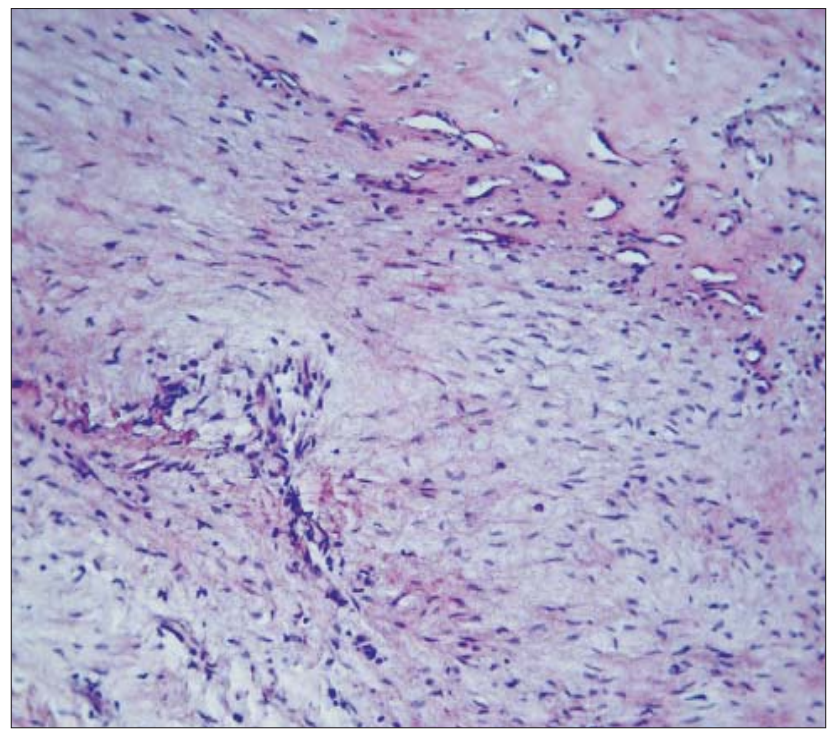

Figure 6 - Fibro-collagenous tissue with an area of granulation tissue (staining: hematoxylin-eosin, lens: $10 \mathrm{X}$ )

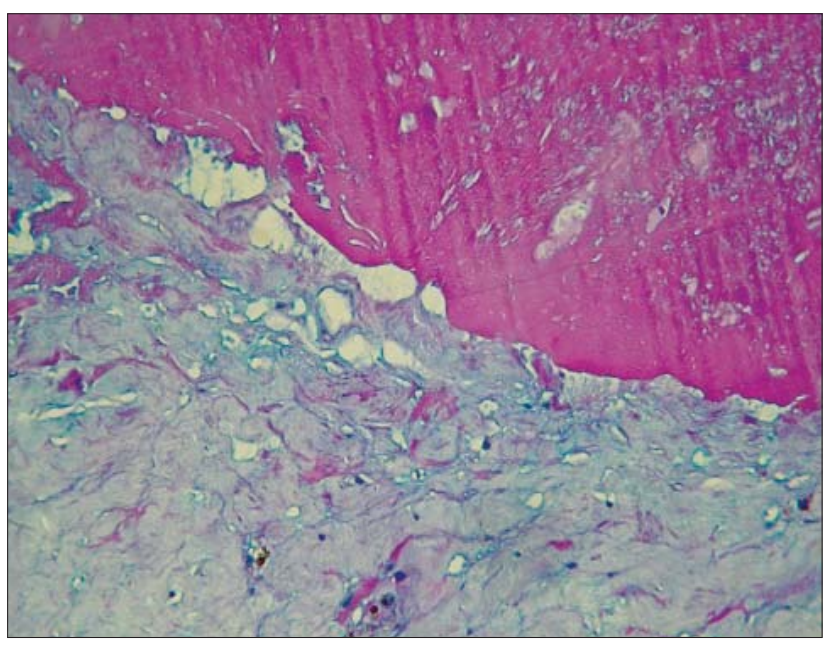

Figure 7 - Fibrin thrombus added over the fibro-collagenous plaque (staining: PAS-Alcian, lens: $20 \mathrm{X}$ ) 
There are several factors that influence the effects of irradiation on arteries, some of them being dependent on the radiotherapeutic technique and others dependent on the patient. The conventional cardiovascular risk factors such as smoking, hypertension, hypercholesterolemia and diabetes act as cofactors, increasing the risk of vascular lesions development after irradiation (8). Data from the literature shows that the younger the patient, the higher the risk of developing radiation arteritis (1), the majority of patients with radiation - induced arterial disease being in their third to fifth decades of life (5). This is also the case of our patient who has several conventional risk factors (smoking, hypertension and dyslipidemia) and developed arterial peripheral disease at a young age with a significant femoral artery stenosis at the irradiation site.

A small irradiated area may allow collateral network to set out, leading to the absence of subjective complaints, while a large one implies fibrosis and arterial stenosis and occlusion, preventing collateral formation and making the injuries symptomatic (8). In this process, the involved region is an important factor as there are radiation sensitive and resistant tissues (2). The consequences of radiation on arteries are technique and dose dependent, peripheral arterial disease being associated with high doses of radiation $(2,5)$. Time is another factor that influences the type of damage caused by radiotherapy; there are early changes that appear within 5 years of irradiation, as arterial rupture or thrombosis $(8,10,11)$, intermediate changes that appear within 10 years of irradiation, as fibrosis, absence of collaterals and occlusion and late changes, after 20 years of irradiation, as local atherosclerosis and periarterial fibrosis (10). In our case, 8 years after radiotherapy, histopathological examination revealed both thrombosis and fibrosis. Also, the irradiated area was relatively small, allowing development of collateral vessels and late occurrence of symptoms.

Conversely, radiotherapy itself represents a risk factor for cardiovascular disease, the relative risk of developing cardiovascular events being up to 5.6 times higher in radiation treated cancer patients than in general population (1).

The characteristics of radiation - induced arterial damage are different from the atherosclerotic ones. There is no manifestation of multi-organ atherosclerosis, radiation arteritis being limited to the site of radiation (12). In patients with testicular tumors, cardiovascular risk increases significantly after 15 years radiation exposure (1). The most commonly radiation affected vessels are the great vessels of the aortic arch, femoral arterial injury being seldom reported and unusual (11). In our case, the occlusions were located on the common, superficial and deep femoral arteries, the last one representing a particular location (11). Besides involvement of the femoral arteries, our patient displayed also bilateral occlusion of the anterior tibial and peroneal arteries, most probably due to atherosclerosis.

In general, the radiation - induced injuries develop insidiously (12), being discovered a few years after radiotherapy (10 years in average, 8 years in our case) $(1,11)$. The effects of radiation can be objectified by stenosis, rupture, dilatation or thrombosis of the vessel (11), the last two being uncommon when it comes to large vessels $(11,13)$. The most common symptom is intermittent claudication or critical limb ischemia, because it is the result of a chronic obliterative process (2). Clinically and angiographically, radiation - induced injury is almost identical to a classic atherosclerosis lesion (7). The angiographic image of radiation arteritis highlights a longer length stenosis than in case of naturally occurring atherosclerotic disease, with focal arterial irregularity, diffuse arterial stenosis or occlusion $(1,7)$. These angiographic features along with clinical history lead to a presumptive diagnosis of radiation arteritis (10).

The management of arterial disease after radiotherapy resembles that for atherosclerotic lesions, depending on anatomy, comorbidities, symptoms, patient's life expectancy and preference $(10,14)$. In order to improve symptoms, a conservative treatment including exercise therapy and pharmacotherapy can be used (14). Because of their antiinflammatory and antithrombotic actions on irradiated endothelial cells, statins may have positive effects on symptoms $(1,2,14)$. There is no indication for prophylactic revascularization in asymptomatic patients $(10,14)$. For those who need revascularization, there are several suitable techniques, from angioplasty and stenting used for focal damage, to bypass surgery for diffuse injuries (10). Choosing between endovascular and surgical revascularization is controversial. The former seems to be superior as the adjacent tissues have a lot of radiation-induced sclerosis (2), but it may cause complications like embolism and restenosis (15). Prophylactic measures needed to limit the extent of radiation arteritis are avoidance of overdoses, a smaller irradiated field and the control of risk factors (11). In our case, surgical revascularization was chosen due to the involvement of deep femoral artery, the patency of this vessel being difficult to obtain by angioplasty. 


\section{CONCLUSIONS}

As radiation - induced arterial disease remains a rarely diagnosed condition, the pathophysiological mechanisms that lead to it remain partly unknown. Arterial damage induced by irradiation appears a few years after cancer therapy, being limited to the exposed field. Inguinal radiotherapy can affect the femoral artery causing a variety of changes as myointimal proliferation, thrombosis, arterial fibrosis, stenosis and accelerated local atherosclerosis. Radiation arteritis is equivalent to accelerated atherosclerosis; thus radiotherapy should be considered as an important risk factor for the development of ischemic syndromes at the irradiated site (2). The management of radiation arteritis remains controversial, every technique having its advantages and disadvantages.

\section{REFERENCES}

1. Gaugler MH, Drouet F, Krempf M. Radiotherapy and atherosclerosis: current data and issues. Med Sci (Paris) 2010;26:740-6.

2. Jurado JA, Bashir R, Burket MW. Radiation-induced peripheral arten disease. Catheter Cardiovasc Interv 2008;72:563-8.

3. Pherwani AD, Reid JA, Keane PF. Synergism between radiotherapy and vascular risk factors in the accelerated development of atherosclerosis. Ann Vasc Surg 2002;16:671-5.

4. Stewart FA, Seemann I, Hoving S, et al. Understanding radiation-induced cardiovascular damage and strategies for intervention. Clin Oncol (R Coll Radiol) 2013;25:617-24.

5. Cavendish JJ, Berman BJ, Schnyder G, et al. Concomitant coronary and multiple arch vessel stenoses in patients treated with external beam radiation: pathophysiological basis and endovascular treatment. Catheter Cardiovasc Interv 2004;62:385-90.

6. Bucci $F$, Robert F, Fiengo L. Radiotherapy-related axillary arteriopathy. Interact Cardiovasc Thorac Surg 2012:15:176-7.

7. Tetik 0 , Yetkin $\mathrm{U}$, Calli $\mathrm{A} 0$ et al. Occlusive arterial disease after radiotherapy for testicular cancer: case report and review of the literature. Vascular 2008;16:239-41.

8. Chuang VP. Radiation-induced arteritis. Seminars in Roentgenology 1994; 29:64-69.

9. Fajardo LF. The pathology of ionizing radiation as defined by morphologic patterns. Acta Oncol 2005:44:13-22.

10. Modrall JG, Sadjadi J. Early and late presentations of radiation arteritis. Semin Vasc Surg 2003:16:209-14.

11. Saliou C, Julia P, Feito B et al. Radiation-induced arterial disease of the lower limb. Ann Vasc Surg 1997;11:173-7.

12. Walker RJ, Wall ML. Accelerated development of mesenteric and renal artery calcific atherosclerosis following radiotherapy for testicular cancer. J Surg Case Rep 2014;2014.

13. Levenback C, Burke TW, Rubin SC, et al. Arterial occlusion complicating treatment of gynecologic cancer: a case series. Gynecol Oncol 1996; 63:40-6.

14. Tendera M, Aboyans V,Bartelink ML, et al. ESC Guidelines on the diagnosis and treatment of peripheral artery diseases. European Heart Journal 2011:32:2851-2906.

15. Bali L, Silhol F, Kateb A, et al. Renal artery stenosis after abdominal radiotherapy. Ann Cardiol Angeiol (Paris) 2009;58:183-6. 